\title{
Subtypes of GABAergic neurons project axons in the neocortex
}

\author{
Shigeyoshi Higo' , Kaori Akashi², Kenji Sakimura ${ }^{2}$ and Nobuaki Tamamaki ${ }^{3 *}$ \\ 1 Kyushu University of Nursing and Social Welfare, Kumamoto, Japan \\ 2 Department of Cellular Neurobiology, Brain Research Institute, Niigata University, Niigata, Japan \\ ${ }^{3}$ Department of Morphological Neural Science, Graduate School of Medical Sciences, Kumamoto University, Kumamoto, Japan
}

\section{Edited by:}

Kathleen S. Rockland, RIKEN Brain

Science Institute, Japan

Reviewed by:

Barbara Clancy, University of Central

Arkansas, USA

Kathleen S. Rockland, RIKEN Brain

Science Institute, Japan

*Correspondence:

Nobuaki Tamamaki, Department of Morphological Neural Science,

Graduate School of Medical Sciences, Kumamoto University, Honjo 1-1-1.

Kumamoto 860-8556, Japan.

e-mail: tamamaki@kumamoto-u.ac.jp $\gamma$-aminobutyric acid (GABA)ergic neurons in the neocortex have been regarded as interneurons and speculated to modulate the activity of neurons locally. Recently, however, several experiments revealed that neuronal nitric oxide synthase (nNOS)-positive GABAergic neurons project corticocortically with long axons. In this study, we illustrate Golgi-like images of the nNOS-positive GABAergic neurons using a nicotinamide adenine dinucleotide phosphate diaphorase (NADPH-d) reaction and follow the emanating axon branches in cat brain sections. These axon branches projected cortico-cortically with other non-labeled arcuate fibers, contra-laterally via the corpus callosum and anterior commissure. The labeled fibers were not limited to the neocortex but found also in the fimbria of the hippocampus. In order to have additional information on these GABAergic neuron projections, we investigated green fluorescent protein (GFP)-labeled GABAergic neurons in GAD67-Cre knock-in/GFP Cre-reporter mice. GFP-labeled axons emanate densely, especially in the fimbria, a small number in the anterior commissure, and very sparsely in the corpus callosum. These two different approaches confirm that not only nNOS-positive GABAergic neurons but also other subtypes of GABAergic neurons project long axons in the cerebral cortex and are in a position to be involved in information processing.

Keywords: GABA, cerebral cortex, nicotinamide adenine dinucleotide phosphate diaphorase, nitric oxide synthase

\section{INTRODUCTION}

GABAergic neurons regulate information processing and are involved in oscillations in the cerebral cortex (Freund and Buzsáki, 1996; Cardin et al., 2009; Sohal et al., 2009). Generally they were thought to be interneurons and act locally. Recently, however, a body of evidence has indicated that GABAergic neurons in the neocortex also project over longer distances. According to our previous report, long-range GABAergic projections originated in layers II, VI and the underlying white matter in mouse neocortex (Tomioka et al., 2005). Several reports have also described the existence of longrange GABAergic projections in the rat's neocortex (Matsubara and Boyd, 1992; McDonald and Burkhalter, 1993). Those projections were not limited to the rodent brain but seemed to exist in feline (Higo et al., 2007) and in primate brain (Barone and Kennedy, 2000; Tomioka and Rockland, 2007).

GABAergic neurons with axons projecting in the ipsilateral hemisphere seem to have similar chemical features and often contain somatostatin (SS)-immunoreactivity (IR) (91\%), neuropeptide Y (NPY)-IR (82\%), and neuronal nitric oxide synthase (nNOS)-IR (71\%) (Tomioka et al., 2005; Higo et al., 2007). Considering these observations, the fact that most nNOS-positive neurons are a subpopulation of SS- and NPY-IR neurons, and nNOS-, NPY-, and SS-triple-positive cells are less than $0.5 \%$ of GABAergic neurons (Kubota et al., 1994; Gonchar and Burkhalter, 1997), it was speculated that the nNOS-positive GABAergic projection neurons compose a very small subpopulation in the neocortical GABAergic neurons. In addition, co-localization of GABA synthesizing enzyme, glutamic acid decarboxylase at $67 \mathrm{~K}$-dalton (GAD67)-IR and a retrograde tracer injected into contralateral hemisphere revealed that another subtype of GABAergic neurons may be involved in the contralateral projection (Gonchar et al., 1995; Kimura and Baughman, 1997; Fabri and Manzoni, 2004). Parvalbumin (PV)-IR GABAergic neurons in the medial septum have been shown to terminate preferentially on hippocampal GABAergic neurons, and act to regulate the activity of pyramidal neurons in the hippocampus (Freund and Antal, 1988). SSIR GABAergic neurons in the hippocampus project back to the medial septum. This circuit was interpreted as the mechanism to cause theta oscillation (Toth et al., 1997). GABAergic projection neurons in the neocortex may act similarly, by inhibiting local GABAergic interneurons and, in turn, allowing cortical principal cell firing.

Although GABAergic projection neurons have been recognized as a subset of neocortical neurons, it is very hard to reveal the projection axons technically. Simple immunohistochemistry of markers like calcium binding proteins or short peptides did not reveal the full axon trajectory from the soma to the terminals. Although nNOS-IR also does not reveal projection axons, NADPH-d reaction reveals nNOS-IR GABAergic neurons in Golgi-like images including their projection fibers (Yan et al., 1996; Barone and Kennedy, 2000; Smiley et al., 2000; Higo et al., 2007). Thus we utilized the NADPH-d reaction to reveal the projection axons of nNOS-IR neurons in the neocortex. In addition, we introduce a new tool, GAD67-Cre knock-in mouse, in which DNA encoding Cre DNA-recombinase in P1 phage was targeted to the locus encoding GAD67. Since the vast majority of GABAergic neurons are expected to be labeled by GFP in the offsprings obtained by mating GAD67-Cre knock-in mouse and GFP Cre-reporter mouse, we expect that all the subtypes of GABAergic projection neurons will be revealed at a glance. 


\section{MATERIALS AND METHODS}

All procedures were carried out according to the guidelines for the care and use of animals approved by the Animal Care and Use Committee at Kumamoto University in accordance with the National Institutes of Health (NIH).

\section{PRODUCTION OF GAD67-Cre KNOCK-IN MOUSE}

The generation of GAD67-Cre knock-in mice will be described into detail elsewhere (Akashi et al. in preparation). To generate the GAD67-knock-in Cre mice, we designed a targeting vector in which Cre recombinase gene was inserted into immediately after the translational initiation site of the GAD67 gene in frame. A knock-in vector pGAD67CreTV contained a $3 \mathrm{~kb}$ fragment at the 5 ' side, a Cre gene placed behind the GAD67 translational start, a $P g k$-neo-p(A) cassette flanked by two Flp recognition target (frt) site, a $7 \mathrm{~kb}$ fragment at the $3^{\prime}$ side, and a MC1 promoter-driven diphtheria toxin gene.

Culture of embryonic stem (ES) cells and generation of chimeric mice were performed as described previously (Kitayama et al., 2001). Briefly, linearized pGAD67CreTV was introduced into C57BL/6 mouse ES cells and then, G418-resistant clones were picked up. Homologous recombined ES clone was identified by Southern blotting. To produce germline chimera, the selected ES cells were microinjected into eight cell-stage embryos of CD-1 mouse strain. The germline chimera of GAD67-Cre mice were crossed with $\mathrm{C} 57 \mathrm{BL} / 6$ mice to generate the GAD67-Cre mice line. Because the knock-out of both GAD67 alleles is lethal at birth (Asada et al., 1997), mice heterozygous for the altered GAD67 allele were used for all the observations in this study. Genotypes were identified by Southern blot hybridization or PCR. Tail genomic DNA was digested with Spe-I or Afl-II and hybridized with $5^{\prime}$ probe or $3^{\prime}$ probe, respectively. PCR was performed with specific three primers. The sequence of each primer and the approximate length of the amplified DNA fragments are described as follows: Gad1 $1^{\text {Cre }}$, g67-2 (5'-TTCCGGAGGTACCACACCTT-3'), g67-5 (5'-TAAGTCGACGCTAGCGAGCGCCTCCCCA-3'), and CreR1 (5'-TTGCCCCTGTTTCACTATCC-3'); wild type, $1.8 \mathrm{kbp}$; mutant: $1.4 \mathrm{kbp}$. The GAD67-Cre knock-in mice were mated with a GFP Cre-reporter mouse (Tanahira et al., 2009). Cre-positive GABAergic neurons are revealed by GFP-expression in the offsprings.

\section{ANIMALS AND RECOVERY OF BRAINS}

In this experiment, three adult cats (2.5- to 4 -kg body weight) and five GAD67-Cre knock-in/GFP Cre-reporter mice were used. Cats were anesthetized with ketamine $(40 \mathrm{mg} / \mathrm{kg}$, i.m. $)$ and Nembutal (50 mg/kg, i.p.). The animals were perfused with phosphate-buffered saline at $\mathrm{pH} 7.4$ (PBS), followed by fixative (4\% paraformaldehyde in $0.1 \mathrm{M}$ phosphate buffer, $\mathrm{pH}$ 7.4) through the left ventricle. Brains were removed, blocked, saturated with a cold solution of $30 \%$ phosphate-buffered sucrose overnight, cut serially on a freezing microtome into $50-\mu \mathrm{m}$ thick coronal sections, and every fifteenth section was collected and used for NADPH-d reaction.

Mice were anesthetized with Nembutal (50 mg/kg i.p.), and perfused with PBS and the same fixative used for cats. Brains were removed, saturated with a cold solution of $30 \%$ phosphate-buffered sucrose overnight, and cut serially on a freezing microtome into $50 \mu \mathrm{m}$ thick coronal sections, and every sixth section was collected and used for GFP-immunohistochemistry or NADPH-d reaction.

\section{VISUALIZATION OF nNOS-POSITIVE NEURONS AND GFP-POSITIVE NEURONS}

The procedure for NADPH-d staining was performed according to the method of Vincent and Kimura (1992). Briefly, floating sections were incubated in a solution of $0.1 \mathrm{M}$ phosphate buffer, $0.3 \%$ Triton $\mathrm{X}-100,0.1 \mathrm{mg} / \mathrm{ml}$ nitroblue tetrazolium, and $1.0 \mathrm{mg} / \mathrm{ml} \beta$-NADPH at $37^{\circ} \mathrm{C}(\mathrm{pH} \mathrm{7.4})$ for $60 \mathrm{~min}$. Sections were rinsed three times in $0.1 \mathrm{M}$ phosphate buffer, $5 \mathrm{~min}$ per rinse, and mounted on gelatinized slides. Sections were then counterstained with $0.3 \%$ Neutral red.

Brain sections of GAD67-Cre knock-in mouse/GFP Cre-reporter mouse were incubated with a primary antibody against GFP, and the immunoreactive site was visualized by $\mathrm{DAB}$ reaction as reported previously (Tamamaki et al., 2000). This procedure allowed us to carry out the morphometry of GFP-positive structures in blightfield microscopy.

\section{COUNTING NADPH-d POSITIVE FIBERS IN THE WHITE MATTER}

To estimate the number of NADPH-d positive fibers in the white matter, we set $200-\mu \mathrm{m}$-site where labeled fibers could be traced as long as possible in the white matter. Then we counted labeled fibers in more than five windows. Since thickness of the sections was $50 \mu \mathrm{m}$, we could estimate the number of labeled fibers contained in $1 \times 10^{4} \mu \mathrm{m}^{2}$ of the cut end of the white matter.

\section{RESULTS}

\section{NADPH-d POSITIVE CELLULAR STRUCTURES IN THE CAT NEOCORTEX}

To reveal axons of nNOS-positive GABAergic neurons, we employed NADPH-d histochemistry in cat cerebral cortex. NADPH-d staining reveals large non-pyramidal neurons (type I) and small nonpyramidal neurons (type II) with dark-blue precipitate in Golgi-like images (Higo et al., 2007) (Figure 1A). nNOS-IR is co-localized with the precipitate in type I cell but not in the type II cells. These two types of NADPH-d-positive cells have been reported in other experimental species (Monkey: Yan and Garey, 1997; Smiley et al., 2000; Rat: Valtschanoff et al., 1993; Kubota et al., 1994) and also in human brain (Judas et al., 1999).

Somata of type I cells are found in the deep layers and white matter of the neocortex (Figures 1A,B). The number of type I cells is highest at the gray matter and white matter boundary. According to the depth in the white matter, the number of type I cells is reduced, and these are almost completely absent near the lateral ventricle (Figure 1B). On the other hand, somata of type II cells are found in the neocortical layers II-VI (Higo et al., 2007). NADPH-d positive cells are also found in the basal ganglia. Since the internal capsule is almost free of NADPH-d positive fibers, the NADPH-d positive fibers and their terminals in the cerebral cortex are assumed to belong to the type I and II NADPH-d positive cells in the cerebral cortex.

NADPH-d positive fibers branch and ramify in the gray matter of the neocortex (Figure 1A). On the other hand, NADPH-d positive thick fibers did not seldom branch in the white matter (Figure 1C). In parallel with the number of type I cells in the white 

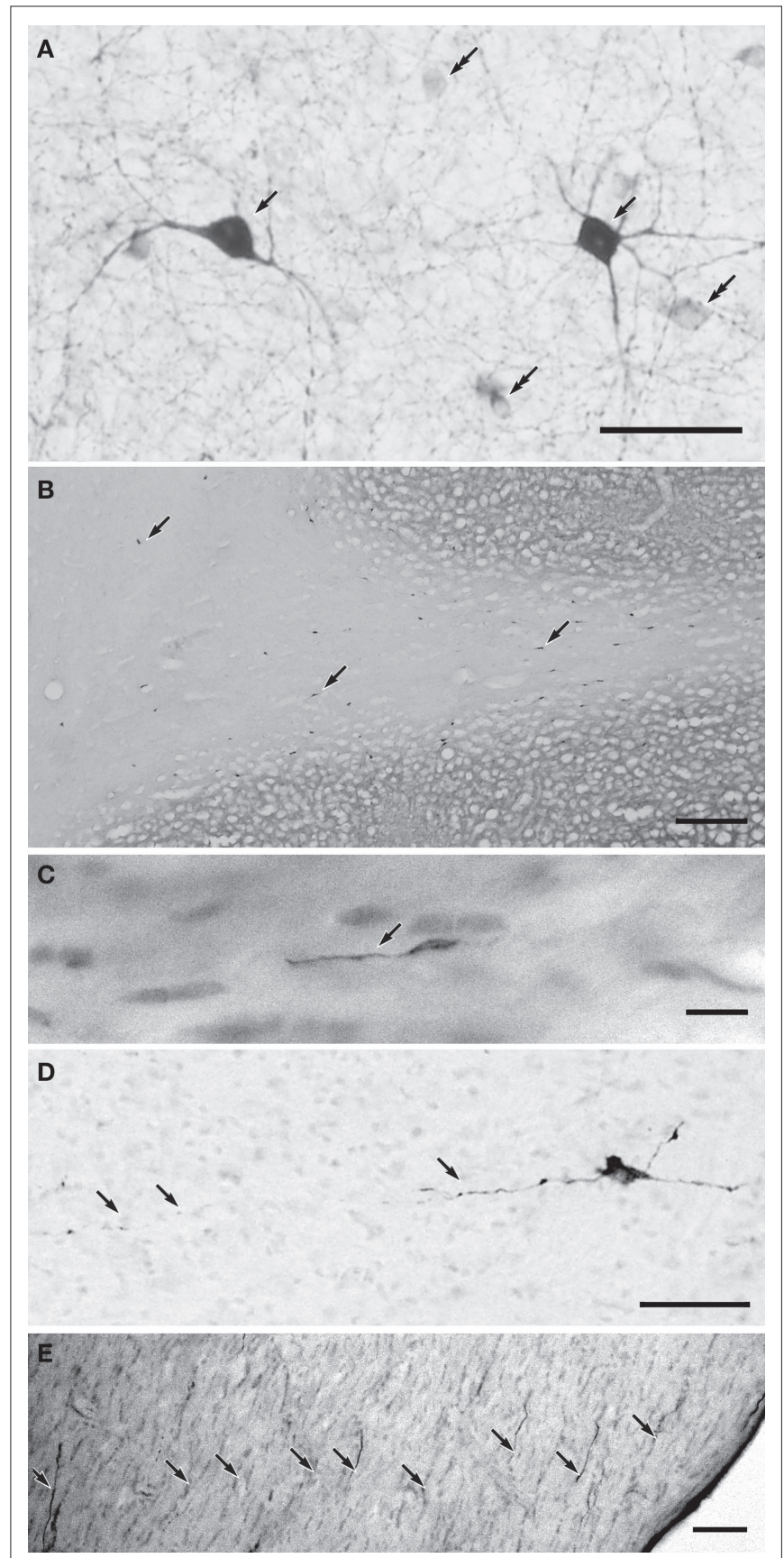

FIGURE 1 | (A) NADPH-d positive GABAergic neurons in the middle suprasylvian gyrus of the cat neocortex. (B) A low magnification photograph of a section with NADPH-d positive neurons in the middle suprasylvian gyrus. (C) A NADPH-d positive axon fiber fragments in the white matter of the neocortex. (D) An NADPH-d-positive type I cell extending an NADPH-d-positive axon fiber in the white matter of the neocortex. (E) NADPH-d positive axon fiber fragments in the fimbria. Arrows indicate NADPH-d positive type I cells and their axons. Double-arrows in (A) indicate NADPH-d type II cells. Calibration bars in (A-E) are $10 \mu \mathrm{m}, 300 \mu \mathrm{m}, 10 \mu \mathrm{m}$, $50 \mu \mathrm{m}$, and $50 \mu \mathrm{m}$, respectively.

matter, the number of NADPH-d positive thick axons also changes: they are highest at the boundary between the gray matter and the white matter and reduced in the deeper white matter. Moreover, large NADPH-d-positive cells originate NADPH-d positive thick axon in the white matter (Figure 1D). The labeled thick axons occur in fascicles and are extended with following the trajectory of other non-labeled axons at every depth of the white matter. We could not find the thick labeled axons giving rise large branches or turning to ascend to the gray matter. We found fragmented thick axons, many in the fimbria $\left(8.8 \pm 2.1 / 10^{4} \mu \mathrm{m}^{2} ; n=5\right)$ (Figure 1E), a small number in the anterior commissure $\left(1.2 \pm 1.2 \mu \mathrm{m}^{2} ; n=5\right)$, and only one in five windows in the corpus callosum $\left(0.2 \pm 0.4 \mu \mathrm{m}^{2} ; n=5\right)$ (see Materials and Methods).

\section{GABAergic PROJECTION AXONS IN THE MOUSE WHITE MATTER}

Three brains were obtained from the mice at 10-weeks old and sectioned into coronal sections at 50- $\mu \mathrm{m}$ thickness. The GFP-positive cells in the mouse brain were distributed similarly to the GFPpositive cells in the GAD67-GFP knock-in mouse (Tamamaki et al., 2003). However, GFP-positive cells in the GAD67-Cre/GFP-reporter mouse are significantly less in number than that in GAD67-GFP mouse. GFP-IR is similar in every GFP-positive cells because the expression of GFP is driven by chick actin (CA)-promoter in all the GFP-positive cells.

Axon fibers with GFP-IR fill the neocortical grey matter, although individual fibers are hard to resolve. GFP-positive axon fibers also occur in the white matter. They can be traced in a few serial sections, but not sufficiently far to determine whether the axon fibers belong to GABAergic projection neurons or not. Therefore, we directed our attention to the fimbria, the anterior commissure, and the corpus callosum. We found a large number of labeled axon segments in the fimbria (Figure 2A). The density of the labeled axons in the fimbria is much higher than that of the NADPH-d positive fibers in the cat fimbria (Figure 1D). A small number of labeled axons traveled in the anterior commissure (Figure 2B), and a few sparsely in the corpus callosum.

The NADPH-d reaction in mouse brain sections does not reveal nNOS-positive neurons in Golgi-like fashion. Although NADPH-d reactions revealed somata and dendrites of type I-like non-pyramidal neurons in mouse neocortex, NADPH-d positive axons appear as dotted-lines and it was difficult to trace their trajectories. The number of labeled axon fragments is countable only in the fimbria. We found similar number of fragmented NADPH-d-labeled axons in the mouse fimbria $\left(3.7 \pm 1.5 / 10^{4} \mu \mathrm{m}^{2} ; n=6\right)$, (Figure $\left.2 \mathrm{C}\right)$ to the case in the cat fimbria.

\section{DISCUSSION}

The present study confirmed the presence of a subpopulation of GABAergic neurons with long projection axons in the cat and mouse neocortex. Moreover, we added the evidence that the GABAergic neurons with long projection axons include subpopulations larger than the nNOS-positive GABAergic neurons, especially in the archicortex.

NADPH-d-positive somata in the white matter originate thick NADPH-d-positive fibers in the white matter of the cat neocortex, which emanate with other non-labeled arcuate fibers, without originating any thick branches, and without bending toward the gray matter of the neocortex. These features may indicate that the total length of NADPH-d-positive fibers in the white matter is long enough to be called as projection fibers, while we regarded 


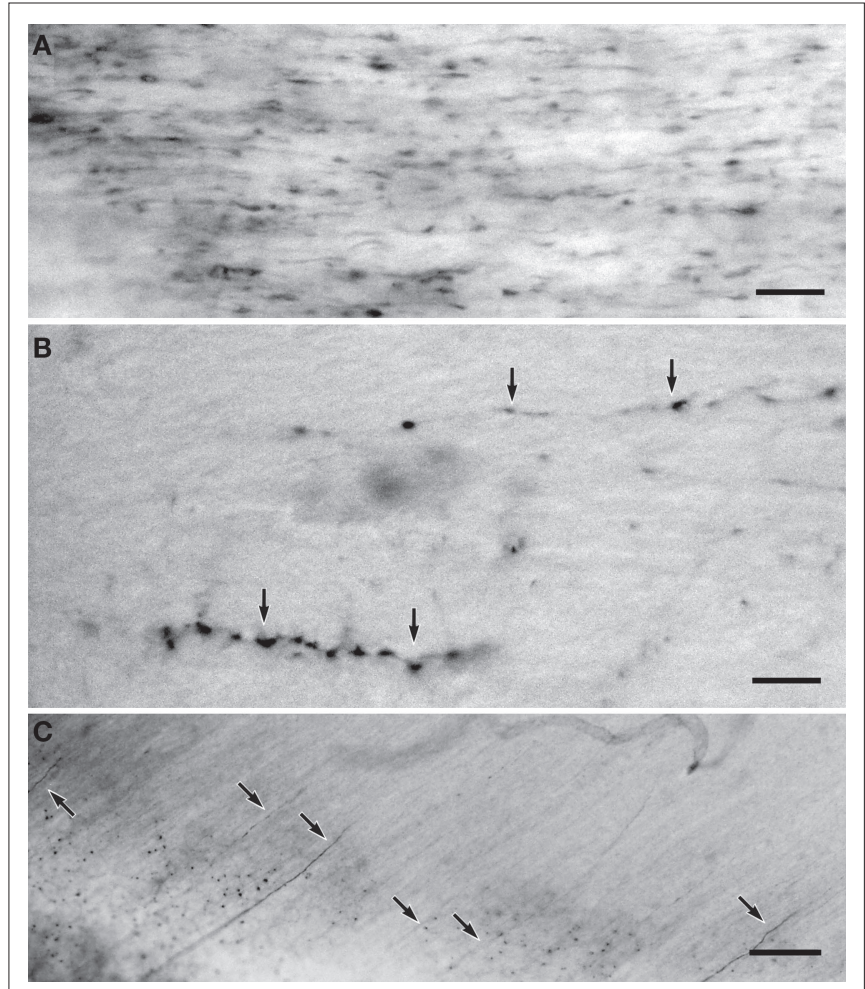

FIGURE 2 | (A) GFP-positive axon fibers in the fimbria of the GAD67-Cre/GFP Cre-reporter mouse brain. (B) GFP-positive axon fibers in the anterior commissure. (C) NADPH-d positive axon fiber fragments in the mouse fimbria. Calibration bar in (A,B) are $10 \mu \mathrm{m}$; calibration bar in (C) is $30 \mu \mathrm{m}$.

GABAergic neuron extending an axon longer than $1.5 \mathrm{~mm}$ from the soma as the GABAergic projection neuron in the mouse (Tomioka et al., 2005). The NADPH-d-positive fibers in the grey matter of the neocortex may include fibers originating from both type I and type II cells.

Formerly, we produced GAD67-GFP knock-in mouse in order to observe GAD67-positive cells and axon fibers traveling in the central nervous system (Tamamaki et al., 2003). The GFP-positive axon fibers were clearly revealed by GFP-fluorescence and could be traced long distances in the brain stem. However, projection axons belonging to the GABAergic neurons in the neocortex seemed to be thin and difficult to identify in the GAD67-GFP mouse. In addition, GAD67-promoter activity seemed to vary in each GABAergic neuron subtype, and the intensity of GFP-fluorescence differed from

\section{REFERENCES}

Asada, H., Kawamura, Y., Maruyama, K., Kume, H., Ding, R. G., Kanbara, N., Kuzume, H., Sanbo, M., Yagi, T., and Obata, K. (1997). Cleft palate and decreased brain gamma-aminobutyric acid in mice lacking the 67-kDa isoform of glutamic acid decarboxylase. Proc. Natl. Acad. Sci. U.S.A. 94, 6496-6499.

Barone, P., and Kennedy, H. (2000). Non-uniformity of neocortex: areal

cell to cell (Tamamaki et al., 2003). With the approach reported here, however, Cre DNA-recombinase deletes floxed stop in the GFP Cre-reporter construct, and GFP was expressed depending on the CA-promoter in the construct (Tanahira et al., 2009). Since Creexpression levels depend on the GAD67-promoter, some subtypes of GABAergic neurons with weak GAD67-promoter activity may fail to delete floxed stop and may not be revealed by GFP-expression. As the result, we may have underestimated the GABAergic neurons projecting contra-laterally via the corpus callosum (Gonchar et al., 1995; Kimura and Baughman, 1997; Fabri and Manzoni, 2004). We feel necessity of introducing additional techniques to estimate the number of GABAergic neurons projecting contra-laterally.

NADPH-d reaction labeled axon fibers of nNOS-positive GABAergic neurons in cats and mice, while GFP-immunohistochemistry in GAD67-Cre/GFP Cre-reporter mouse labeled both projection fibers originating in nNOS-positive GABAergic neurons and those in the other subtypes of GABAergic neurons. However, the distribution pattern of the labeled axon fragments was similar in both preparations. NADPH-d reactive axons and GFP-IR axons were found to be very sparse in the corpus callosum, sparsely in the anterior commissure, and many in the fimbria. Labeled fibers in the fimbria will include afferent- and efferent-fibers from and to the subcortical nuclei, in addition to the commissural fibers through the ventral hippocampal commissure. Excluding subcortical afferent- and efferent-fibers, we speculate that the GABAergic commissural fibers reciprocally interconnect archi- and paleo-cortex.

When GABAergic projection neurons terminate on GABAergic interneurons, they will inhibit the interneurons and thus disinhibit excitatory principle neurons in the hippocampus (Freund and Antal, 1988; Toth et al., 1997). Although NADPH-d positive fibers in cat neocortex ramified and seemed to terminate on many types of neurons (i.e. both principle neurons and inhibitory neurons), $\mathrm{NADPH}$-d-positive fibers originating in the type I cells may innervate GABAergic neurons preferentially. To address this possibility, we plan to investigate the terminal of labeled nNOS-positive axon fibers in future. One of the important results elucidated in this study is that a large number of GABAergic neurons are also involved in the cortico-fugal, cortico-cortical, and callosal projections. Each subtype of GABAergic projection neuron may contribute to information processing in the neocortex in different ways.

\section{ACKNOWLEDGMENTS}

This work was supported by Grants-in-Aids from the Ministry of Education, Science, Sports and Culture of Japan to K. Sakimura and N. Tamamaki.

immunoreactivity in callosal projecting neurons of cat and rat somatic sensory areas. Neuroscience 123, $557-566$.

Freund, T.F., and Antal, M. (1988). GABAcontaining neurons in the septum control inhibitory interneurons in the hippocampus. Nature 336, 170-173.

Freund, T. F., and Buzsáki, G. (1996). Interneurons of the hippocampus. Hippocampus 6, 347-470.
Gonchar, Y., and Burkhalter, A. (1997). Three distinct families of GABAergic neurons in rat visual cortex. Cereb. Cortex 7, 347-358.

Gonchar, Y. A., Johnson, P. B., and Weinberg, R. J. (1995). GABA-immunopositive neurons in rat neocortex with contralateral projections to S-I. Brain Res. 697, 27-34.

Higo, S., Udaka, N., and Tamamaki, N. (2007). Long-range GABAergic 
projection neurons in the cat neocortex. J. Comp. Neurol. 503, 421-431.

Judas, M., Sestan, N., and Kostović, I. (1999). Nitrinergic neurons in the developing and adult human telencephalon: Transient and permanent patterns of expression in comparison to other mammals. Microsc. Res. Tech. $45,401-419$.

Kimura, F., and Baughman, R. W. (1997). GABAergic transcallosal neurons in developing rat neocortex. Eur. J. Neurosci. 9, 1137-1143.

Kitayama, K., Abe, M., Kakizaki, T., Honma, ., Natsume, R., Fukaya, M., Watanabe,M.,Miyazaki,J.,Mishina,M., and Sakimura, K. (2001). Purkinje cellspecific and inducible gene recombination system generated from C57BL/6 mouse ES cells. Biochem. Biophys. Res. Commun. 281, 1134-1140.

Kubota, Y., Hattori, R., and Yui, Y. (1994). Three distinct subpopulation of GABAergic neurons in rat frontal agranular cortex. Brain Res. 649, 159-173.

Matsubara, J. A., and Boyd, J. D. (1992). Presence of GABA-immunoreactive neurons within intracortical patches in area 18 of the cat. Brain Res. 583, 161-170.

McDonald,C.T., and Burkhalter,A. (1993). Organization of long-range inhibitory connections within rat visual cortex J. Neruosci. 13, 768-781.

Smiley,J.F., McGinnis, J.P., and Javitt, D. C. (2000). Nitric oxide synthase interneurons in the monkey cerebral cortex are subsets of the somatostatin, neuropeptide $\mathrm{Y}$, and calbindin cells. Brain Res. 863, 205-212.

Sohal, V. S., Zhang, F., Yizhar, O., and Deisseroth, K. (2009). Parvalbumin neurons and gamma rhythms enhance cortical circuit performance. Nature 459, 698-702.

Tamamaki, N., Nakamura, K., Furuta, T., Asamoto, K., and Kaneko, T. (2000). Neurons in Golgi-stain-like images revealed by GFP-adenovirus infection in vivo. Neurosci. Res. 38, 231-236.

Tamamaki, N., Yanagawa, Y., Tomioka, R., Miyazaki, J., Obata, K., and Kaneko, T. (2003). Green fluorescent protein expression and colocalization with calretinin, parvalbumin, and somatostatin in the GAD67-GFP knock-in mouse. J. Comp. Neurol. 467, 60-79.

Tanahira, C., Higo, S., Watanabe, K., Tomioka, R., Ebihara, S., Kaneko, T., and Tamamaki,N.(2009).Parvalbumin neurons in the forebrain as revealed by parvalbumin-Cre transgenic mice. Neurosci. Res. 63, 213-223.
Tomioka, R., Okamoto, K., Furuta, T., Fujiyama, F., Iwasato, T., Yanagawa, Y., Obata,K.,Kaneko, T., and Tamamaki, N (2005). Demonstration of long-range GABAergic connections distributed throughout the mouse neocortex. Eur. J. Neurosci. 21, 1587-1600.

Tomioka, R., and Rockland K. S. (2007). Long-distance corticocortical GABAergic neurons in the adult monkey white and gray matter. J. Comp. Neurol. 505, 526-538.

Toth, K., Freund, T. F., and Miles, R. (1997). Disinhibition of rat hippocampal pyramidal cells by GABAergic afferents from the septum. J. Physiol. (Lond.) 500, 463-474.

Valtschanoff, J. G., Weinberg, R. J., Kharazia, V. N., Schmidt, H. H., Nakane, M., and Rustioni, A. (1993). Neurons in rat cerebral cortex that synthesize nitric oxide: NADPHdiaphorase histochemistry, NOS immunocytochemistry, and colocalization with GABA. Neurosci. Lett.157, 157-161.

Vincent, S. R., and Kimura, H. (1992). Histochemical mapping of nitric oxide synthase in the rat brain. Neuroscience $46,755-784$.

Yan, X. X., and Garey, L. J. (1997). Morphological diversity of nitric oxide synthesizing neurons in mammalian cerebral cortex. J. Hirnforsch. 38, 165-172.

Yan, X. X., Jen, L. S., and Garey, L. J. (1996). NADPH-diaphorase-positive neurons in primate cerebral cortex colocalize with GABA and calcium-binding proteins. Cereb. Cortex 6, 524-529.

Conflict of Interest Statement: The authors declare that the research was conducted in the absence of any commercial or financial relationships that could be construed as a potential conflict of interest.

Received: 13 July 2009; paperpending published: 27 July 2009; accepted: 15 October 2009; published online: 09 November 2009.

Citation: Higo S, Akashi K, Sakimura K and Tamamaki $N$ (2009) Subtypes of GABAergic neurons project axons in the neocortex. Front. Neuroanat. 3:25. doi: 10.3389/neuro.05.025.2009

Copyright $\odot 2009$ Higo, Akashi, Sakimura and Tamamaki. This is an open-access article subject to an exclusive license agreement between the authors and the Frontiers Research Foundation, which permits unrestricted use, distribution, and reproduction in any medium, provided the original authors and source are credited. 